\title{
P-toluene Sulfonic Acid Catalyst in Synthesis of Ethylene Glycol Butyl Ether Acetate
}

\author{
Gengqiang Zhang, Shiyao Zhu, Wenzhu Zhang, Yongjie Wang \\ Liaoyang Campus, Shenyang University of Technology \\ School of Petrochemical Engineering, Liaoyang 111003, China \\ wyjsgd@qq.com
}

\begin{abstract}
Ethylene glycol butyl ether and acetic acid as raw materials, cyclohexane as dehydrant, by direct esterification method, under p-toluene sulfonic acid catalyst, the catalytic synthesis of ethylene glycol butyl ether acetate(EGBEA). In the single factor experiment examines the usage of the influence of the reaction, and through the orthogonal experiment of catalyst, material ratio, reaction time is discussed such as the influence of factors on the reaction. Using infrared spectroscopy, the products were characterized. Results show that when the ethylene glycol butyl ether is $11.817 \mathrm{~g}$, acetic acid is $7.806 \mathrm{~g}$, P-toluene sulfonic acid catalyst is $3 \%$, cyclohexane as $23.340 \mathrm{~g}$, Keep the return rate $2 \sim 3 \mathrm{~d} / \mathrm{s}$, speed $3 \sim 4 \mathrm{r} / \mathrm{s}$, reflux temperature $80 \sim 95{ }^{\circ} \mathrm{C}$. Constantly in the process of reaction 60 minutes (reaction zone was born into the water), atmospheric distillation cyclohexane, before the rectification products, collection of 191 193 ${ }^{\circ} \mathrm{C}$ fractions. Vested colorless transparent EGBEA, the yield is $98.81 \%$. The bullishness of chromatographic analysis is more than $99 \%$ purity.
\end{abstract}

Keywords- esterification; catalyst; p-toluene sulfonic acid; ethylene glycol butyl ether; ester

\section{INTRODUCTION}

Ethylene glycol butyl ether acetate (EGMBA) acetate, also known as Ding Jirong fiber agent is a kind of high boiling point, containing more than one of functionality, glycol butyl ether ester solvent, can be used as the coalescing aid of emulsioni paint, it dissolves on a variety of paint has excellent performance, making it in colorful coating and emulsion coatings obtained widespread application in[1, 2].Now the main production methods of alcohol ether acetate in alcohol ether and ice acetic acid as the raw material of direct esterification method[3], the method is relatively mature and reliable. Most of the traditional process using sulfuric acid as catalyst, the downside is side reaction, easy equipment corrosion, large amount of waste water, easy to cause environmental pollution[4].

In the ethylene glycol butyl ether and ice acetic acid as raw materials and p-toluene sulfonic acid as catalyst, the catalytic synthesis of ethylene glycol butyl ether acetate, effect is good. The process of equipment corrosion degree is small, basic don't cause pollution to the environment, simple post-treatment processing, shorter reaction time, the yield is higher also. Ethylene glycol butyl ether and acetic acid, esterification in the presence of acidic catalyst, the first is by and $\mathrm{C}=\mathrm{O}$ double oxygen, reduce the electron cloud density of carbon atoms of carboxyl, and ethylene glycol butyl ether molecules attack after electron cloud density low carbon atoms, the formation of ethylene glycol butyl ether and the intermediate of acetic acid, then the intermediate lose a molecule of water, again lose a, the resulting glycol butyl ether acetate[5-11].

A EXPERIMENTAL

Reagent and instrument

Ethylene glycol butyl ether (Shenyang dongling, fine chemical company), acetic acid in fine chemical industry co., LTD. (tianjin), cyclohexane fine chemical co., LTD. (Tianjin), p-toluene sulfonic acid (Shenyang chemical reagent factory), all reagents were analytical pure. Nicolet 5700 infrared spectrometer (USA), GC9790, WAY-2W abbe refractometer, SHT digital display temperature of magnetic stirring electric.

\section{SELECTION OF CATALYSTS FOR SINGLE FACTOR}

\section{EXPERIMENT}

Catalyst can reduce activation energy and increase the rate of reaction, has much effect on the chemical reaction. Common catalysts for esterification are acid(H2SO4, $\mathrm{H} 3 \mathrm{PO} 4, \mathrm{C} 6 \mathrm{H} 4 \mathrm{SO} 3 \mathrm{H}$, etc.), anhydrous acid salt ( $\mathrm{AlCl} 3$, $\mathrm{KHSO} 4$, etc.), oxide (A12O3, $\mathrm{SiO} 2$, etc.), strongly acidic cationic exchange resin, zeolite.

Using single factor experiment method, use the same water, the ratio of raw material constant, catalyst, reaction time constant, etc., the same reaction conditions, discusses the aminobenzene sulfonic acid, p-toluene sulfonic acid, stannous chloride, phosphotungstic acid, phosphor molybdate, silicon tungsten acid, cerium sulfate, etc. of ethylene glycol butyl ether and acetic acid catalytic synthesis of ethylene glycol butyl ether acetate yield effect. Feeding is: acetic acid 6.005 g, ethylene glycol butyl ether, $11.817 \mathrm{~g}$, catalyst $0.446 \mathrm{~g}$, dehydrant(cyclohexane) dosage $30 \mathrm{ml}$, reaction time of 60 minutes, reflux temperature 80 950C. Experimental results are shown in table 1. 
TABLE I INTENSITY OF DIFFERENT ACID CATALYSTS ON THE YIELD OF EGBEA

\begin{tabular}{ccc}
\hline Catalyst type & Acid intensity $(0.1 \mathrm{~mol} / \mathrm{L} \mathrm{pH}$ value $)$ & EGBEA yield $/ \%$ \\
\hline Blank test & & 5.16 \\
Aminobenzene sulfonic acid & 1.92 & 15.90 \\
Stannous chloride & 1.51 & 48.67 \\
Phosphomolybdic acid & $\mathrm{PH}=2.65(0.1 \%)$ aqueous solution & 75.11 \\
Silicotungstic acid & $\mathrm{PH}=2.85(0.1 \%)$ aqueous solution & 80.17 \\
Phosphotungstic acid & $\mathrm{PH}=2.74(0.1 \%)$ aqueous solution & 92.56 \\
P-toluene sulfonic acid & 1.01 & 93.67 \\
Titanium sulfate & 0.84 & 89.89 \\
Sulfuric acid & 0.84 & 74.08 \\
\hline
\end{tabular}

Experimental results are shown in table 1, from table 1 shows that when the dosage of catalyst phase at the same time, the esterification reaction yield sequence of p-toluene sulfonic acid $>$ phosphotungstic acid $>$ titanium sulfate $>$ silicotungstic acid $>$ phosphomolybdic acid $>$ sulfuric acid $>$ stannous chloride $>$ aminobenzene sulfonic acid $>$ blank test, p-toluene sulfonic acid as catalyst, the yield of EGBEA is $93.67 \%$; P-toluene sulfonic acid has a strong acid, catalyst effect is good, stable property, strong water imbibition and no oxidation, advantages of carbonation weaker, so choose p-toluene sulfonic acid catalyst for this reaction.

\section{CHOICE OF SINGLE FACTOR EXPERIMENT DEHYDRANT}

Using single factor experiment method, dosage of water remains unchanged, the ratio of raw material constant, catalyst, reaction time constant, join in the reactor cyclohexane, n-hexane, petroleum ether, benzene, toluene, xylene, examines the types of water influencing on the yield of EGBEA. Feeding is: acetic acid $7.806 \mathrm{~g}$, ethylene glycol butyl ether, $11.817 \mathrm{~g}$, catalyst $0.491 \mathrm{~g}, 30$ $\mathrm{ml}$ water dosage, reaction time 60 minutes, reflux temperature $80 \sim 95{ }^{0} \mathrm{C}$. The experimental results are shown in table 2 .

TABLE II DIFFERENT USAGE OF INFLUENCING ON THE YIELD OF EGBEA

\begin{tabular}{cc}
\hline Dehydrant & EGBEA yield / \% \\
\hline Benzene & 63.47 \\
Toluene & 57.88 \\
Xylene & 57.85 \\
Petroleum ether & 74.37 \\
n-Hexane & 67.56 \\
Cyclohexane & 76.89 \\
\hline
\end{tabular}

Can be seen from the data in table 2 , when the water is cyclohexane products the highest yield(76.89\%), low cyclohexane boiling point, low toxicity, low pollution to the environment, after the reaction is easy separation, and benzene, toluene, xylene, etc have certain toxicity, environmental pollution, so the selection of cyclohexane dehydrant for this reaction.

TABLE III FACTORS AND LEVELS ORTHOGONAL EXPERIMENT

\begin{tabular}{cccc}
\hline level & A mole ratio of reactants & $\begin{array}{c}\text { factors } \\
\text { B catalyst } / \%\end{array}$ & C time $/$ mins \\
\hline 1 & $1: 1.1$ & 2.0 & 50 \\
2 & $1: 1.2$ & 2.5 & 60 \\
3 & $1: 1.3$ & 3.0 & 70 \\
\hline
\end{tabular}




\section{DETERMINATION OF ORTHOGONAL TEST OF OPTIMAL REACTION CONDITIONS}

P-toluene sulfonic acid as catalyst, reaction ratio of raw materials (molar ratio of ethylene glycol butyl ether and acetic acid) and reaction time as factors, do the three factors three levels orthogonal experiment. Factors and levels shown in table 3 , results and analysis are shown in table 4.

$\mathrm{R}$ corresponding factors are used to measure the size of the experiment, the size of the poor big factors, conversion rates caused by the impact is bigger, often is the important factor, and poor little factors often is not important. By the experimental data in table 4 shows: in the first column of the mole ratio of reactants is poor, the biggest for $\mathrm{R} 1=8.70$, shows that for ester yield, order to the importance of the three factors: reaction time $<$ the dosage of the catalyst $<$ mole ratio of reactants. $\mathrm{K}$ value can be obtained from table 4 , the theory of optimum technological conditions for, mole ratio of $1: 1.3$, catalyst $3 \%$, the reaction time of 60 minutes, acetic acid conversion rate of $97.23 \%$, the $3,3,2$ is best.

TABLE IV EGBEA SYNTHESIS REACTION OF ORTHOGONAL EXPERIMENT DATA

\begin{tabular}{ccccc}
\hline No. & A & B & C & EGBEA yield / \% \\
\hline 1 & 1 & 1 & 1 & 94.00 \\
2 & 2 & 2 & 2 & 95.15 \\
3 & 3 & 3 & 3 & 97.39 \\
4 & 1 & 2 & 3 & 91.97 \\
5 & 2 & 3 & 1 & 94.88 \\
6 & 3 & 1 & 2 & 97.23 \\
7 & 1 & 3 & 2 & 95.34 \\
8 & 2 & 1 & 3 & 95.77 \\
9 & 3 & 2 & 1 & 95.39 \\
$\mathrm{~K}_{1}$ & 281.31 & 287.00 & 284.27 & $\mathrm{R}_{1}=8.70$ \\
$\mathrm{~K}_{2}$ & 285.80 & 282.51 & 287.72 & $\mathrm{R}_{2}=5.10$ \\
$\mathrm{~K}_{3}$ & 290.01 & 287.61 & 285.13 & $\mathrm{R}_{3}=3.45$ \\
\hline
\end{tabular}

\section{VALIDATION TEST UNDER THE OPTIMAL CONDITIONS}

P-toluene sulfonic acid $3 \%$, acetic acid $7.806 \mathrm{~g}$, ethylene glycol butyl ether $11.817 \mathrm{~g}$, and cyclohexane $23.340 \mathrm{~g}$, in turn, add to the four flask, fitting of reflux device, on a magnetic stirring electric heating, heating reflux. Keep the return rate $2 \sim 3 \mathrm{~d} / \mathrm{s}$, speed $3 \sim 4 \mathrm{r} / \mathrm{s}$, reflux temperature $80 \sim 95{ }^{\circ} \mathrm{C}$. Constantly in the process of reaction 60 minutes (reaction zone was born into the water), atmospheric distillation cyclohexane, before the rectification products, collection of 191 193 ${ }^{\circ} \mathrm{C}$ fractions. Vested colorless transparent EGBEA, the yield is $98.81 \%$.

\section{RESULTS AND DISCUSSION}

\section{Quantitative analysis of EGBEA}

Product bullishness of chromatographic quantitative analysis, analysis of the conditions for OV-101, capillary column $(30 \mathrm{~m} \times 0.25 \mathrm{~mm})$, column temperature of $170{ }^{\circ} \mathrm{C}$, time 10 minutes, vaporizing chamber temperature $170{ }^{\circ} \mathrm{C}$, detector temperature $170{ }^{\circ} \mathrm{C}$. Hydrogen flame detector, carrier gas $\mathrm{N} 2$, flow of $15 \mathrm{ml} / \mathrm{s}$, quantitatively by area normalization method. Products by the analysis of purity is more than $99.0 \%$.

2 Infrared spectrum analysis of EGBEA

Infrared spectrogram analysis: $1237.48 \mathrm{~cm}-1-\mathrm{C}-\mathrm{O}$ stretching vibration, $1200-1600 \mathrm{~cm}-1$ for carbon chain frame vibration, $1742.17 \mathrm{~cm}-1$ for saturated carboxylic acid alkyl ester vibration, the 3000-2800 cm-1 -C-H stretching vibration; Due to no hydroxyl in the spectrogram, therefore, known product of EGBEA.

3 Determination of refractive index

Abbe refractometer WYA-2 under $25^{\circ} \mathrm{C}$ determination of EGBEA of refractive index is 1.4892 1.4896. CONCLUSION

P-toluene sulfonic acid as catalyst, ethylene glycol butyl ether, acetic acid as raw materials, cyclohexane as dehydrant, through direct catalytic esterification optimum reaction conditions for synthesis of EGBEA as catalyst dosage of p-toluene sulfonic acid is $3 \%$, ethylene glycol butyl ether dosage is $11.817 \mathrm{~g}$, acetic acid dosage is 7.806 $\mathrm{g}$, dehydrant cyclohexane dosage is $23.340 \mathrm{~g}$, the reaction time for 60 minutes, the reaction temperature is $80 \sim 95{ }^{\circ} \mathrm{C}$. Under this condition, the yield of EGBEA is $98.81 \%$, the refractive index is 1.4892 1.4896. The bullishness of chromatographic analysis is more than $99 \%$ purity.

\section{REFERENCE}

[1] Yanhui Qiao, Junjiang Teng, Liqun Ma, etal., "Synthesis of 2-Butoxyethyl Acetate Catalyzed by Ionic Liquid of 1-Methyl-3-Butylimidazolium Hydrosulfate," Chemical Industry and Engineering Progress, vol. 30, June. 2011, pp1347-1350.

[2] Xun Zhu, "Study on Improvement of Preparation of Ethyleneglycol Monoethyl Ether Acetate," Applied Chemical Industry, vol.35, April. 2006, pp298-299. 
[3] Jie Chen, Jianchun Jiang, Junming Xu, "Recent Research of Solid Acid Catalysts Used in Esterification," Advances in Fine Petrochemicals, vol. 10, March. 2009, pp32-36.

[4] Longrui Qian, "High Performance Industrial Solvent Ethyleneglycol Monoalkyl Ether Carboxylates," Fine Petrochemicals, vol. 8, March. 1991, pp60-64.

[5] Yongjie Wang, Shiyao Zhu, Gengqiang Zhang, "Selective Esterification of Ethylene Glycol Ethyl Ether Over Silica-cerous," Applied Mechanics and Materials. vols. 651-633, July. 2014, pp88-91.

[6] Guozhen Gong, Xuezheng Liang, Jian Wang, etal., "Synthesis of 2-Methoxyethyl Acetate by Transesterification on the Solid Base K2CO3/A12O3," Fine Chemicals, vol. 25, October. 2008, pp982-984.
[7] Jianhua Li, "Study on Synthesis of Diethylene Glycol Monobutyl Ether Acetate by Strongly Acidic Cationic Exchange Resin as Catalyst," Advances in Fine Petrochemicals, vol. 12, March. 2011, pp17-20.

[8] Dengfeng Guo, Hong Liu, Zhun Liu, etal., "Synthesis of Ethylene Glycol Monobutyl Ether with KF/A12O3 Solid Base Catalyst,' Journal of Nanjing University of Science and Technology(Natural Science), vol. 35, January. 2011, pp122-126.

[9] Junjiang Teng, Chunhai Li, Liqun Ma, etal., "Synthesis of 2-butoxyethyl acetate in the presence of ionic liquid catalyst," Chemical Research, vol. 22, June. 2011, pp46-48.

[10] Zuojun Wei, Yan Li, Feijin Li, etal., "Catalytic Esterification Reactions over Immobilized Brpnsted Ionic Liquid," CIESC Journal, vol. 60, June. 2009, pp1452-1458. 